\title{
Type 2 diabetes mellitus and increased risk of substance use disorder development: an educational module to promote assessment implementation in endocrinological settings
}

\author{
Olivia Sosnoski \\ Western Connecticut State University \\ M.S. Addiction Studies \\ PSY 590 \\ Supervised by: Dr. J. Wyatt \\ May, 2021
}




\section{Foreword}

I'd like to thank Dr. Laura Nally, my original research mentor and the woman who helped me discover my passion for clinical research. I'd also like to thank Dr. Janan Wyatt, who has so generously given me her time, perspective, and knowledge on this research project. Dr. Wyatt-your help is so greatly appreciated and words cannot express the amount of value you have added to both my master's education and my future in research.

Thank you. 


\section{Literature Review}

\section{Substance Use Disorder Overview}

Substance use disorders (hence forth referred to as SUDs) are a broad category of psychiatric diseases that include dependence on a range of substances. From the DSM-5, it is noted that all drugs - regardless of what kind - share a common direct activation of the brain reward system when consumed in excess (American Psychiatric Association, 2013). It is this reward system activation that reinforces behaviors and memories associated with substance use (American Psychiatric Association, 2013). This intense activation of the reward system can lead to significant impairment and distress among substance users resulting from the neglect of normal activities (American Psychiatric Association, 2013). While there are an abundance of substances that may result in the development of a SUD, this study will focus on alcohol use disorder (hence forth referred to as AUD), tobacco use disorder (hence forth referred to as TUD), opioid use disorder (hence forth referred to as OUD), cocaine use disorder (hence forth referred to as CUD). These specific substance use disorders were selected based upon their prevalence, however, it is noteworthy that virtually any substance can result in a use disorder.

Across the board, significant associations have been found between depression and the presence of SUDs, with odds ratios falling between 1.3 and 2.6 (Iqbal et al., 2019). This connection between depression and SUDs may arise as early as adolescence, as the likelihood of SUD development is increased among adolescents with depression (Iqbal et al., 2019).

Furthermore, populations with dually-diagnosed depression and SUDs have been noted to have significantly worse clinical outcomes as compared to patients with just one psychiatric diagnosis (Iqbal et al., 2019). 
A systematic review and meta-analysis was done combining data from community and clinical settings from 1990 through 2019 (Hunt et al., 2020). The total sample size included 348,550 subjects and found that the prevalence of any SUD among adults with major depressive disorder was $25 \%$ (Hunt et al., 2020). Of the substances surveyed, it was found that AUD had the highest prevalence at $20.8 \%$, and that the combination of AUD and major depressive disorder in men was even higher at 36\%, compared to just $19 \%$ for women (Hunt et al., 2020).

Specifically pertaining to AUD, its been found that chronic, excessive consumption of alcohol can result in a pro-inflammatory state with the release of cytokines and neuroimmune mediators (Neupane, 2016). This immune response can alter brain function and may also impact microglia in the central nervous system, encouraging the development and progression of depressive illness (Neupane, 2016). The inflammatory nature of this interaction may account for the high life-time comorbidity rate between major depression and AUD, which is an astoundingly high $20.5 \%$ (Neupane, 2016).

Anxiety is an additional psychiatric disorder to consider in the context of SUDs. There are high comorbidity rates between anxiety disorders and substance use disorders, with lifetime prevalence of co-morbid anxiety and SUD of $2 \%$ among the general population (Smith and Book, 2008). Of the various types of anxiety disorders, generalized anxiety disorder was found to be the most common co-morbid anxiety disorder with SUDs (Smith and Book, 2008). When considering clinical populations with the aforementioned depressive disorders and/or anxiety disorders, it becomes apparent that populations with these co-occurring psychiatric disorders are at increased vulnerability for SUD development.

While the dual relationship between psychiatric disorders and SUDs are apparent, there is often a third confounding variable that may be overlooked: the presence of physiological 
disorders and chronic illness. Early research regarding SUDs and physiological conditions highlighted increased SUD rates in clinical populations with arthritis, heart disease, chronic lung disease, and high blood pressure (Wells et al., 1989). Unfortunately, this literature oversaw diabetes as a risk factor associated with substance use disorder development and failed to consider that the increased rates of psychiatric disorders in diabetic adults may also play a role in SUD development (Wells et al., 1989). This early information is especially misleading, because type 2 diabetes mellitus (hence forth referred to as: T2DM) affects $9 \%$ of adults (Wu et al., 2015) and can leave clinical populations exceptionally vulnerable to SUD development. Among adults with high-risk type 2 diabetes, a study found that $48 \%$ of patients had a SUD recorded in their medical records, while $75 \%$ of the same clinical population with high risk T2DM had a record of other mental health disorders (Wu et al., 2015).

\section{T2DM \& SUD}

While the above explored psychiatric risk-factors that may be associated with the development of SUDs in adults with T2DM, further research has been done on the direct relationship between T2DM and SUDs. One review, which described the relationship between T2DM and SUDs as being "understudied," noted that NUD was particularly prominent among adults with T2DM with a prevalence ranging from 10-26\% (Walter et al., 2017). A more recent study, which analyzed 17 years of patient data, found the following SUD prevalence rates among 50,897 adults with T2DM: $21.8 \%$ of diabetics were diagnosed with TUD, $8.0 \%$ of diabetics were diagnosed with AUD, $2.2 \%$ of diabetics were diagnosed with CUD, and $1.9 \%$ of diabetics were diagnosed with OUD (Winhusen et al., 2019). In comparison to a recent SAMHSA trend report on SUDs in adults ages $18+$, the prevalence of AUD in the general population was $6.8 \%$, CUD 
was $0.4 \%$, and combined RX pain relievers (opioids) and heroin was $0.9 \%$ (Lipari and Van Horn, 2017). While the SAMSHA report did not cover NUD rates, it's noteworthy that the T2DM clinical population had higher prevalence rates of AUD, CUD, and OUD than the general population. Furthermore, the increases in OUD and CUD rates among the T2DM population were drastic, with the OUD prevalence doubled in diabetics and CUD prevalence in diabetics being more than five times higher than the general population.

A 2016 meta-analysis examined the co-occurrence of AUD and T2DM, and suggested that the prevalence of AUD may be even higher than the aforementioned 8.0\% (Vancampfort et al., 2016). This meta-analysis adjusted the collection of data to population-based studies, and found the prevalence of T2DM among those with AUD to be 9.9\%, and that a history of AUD increased the risk of T2DM development compared to the general population (Vancampfort et al., 2016). This data is particularly profound when considering that this population has higher risk of psychiatric disorders such as depression and anxiety, which is associated with both T2DM and substance use disorder risk. This meta-analysis also may pose a dual relationship between SUDs and T2DM, where the presence of either condition first consequently increases the risk of developing the other condition.

\section{T2DM \& Psychiatric Risk Factors}

In 2013, one study found the prevalence of depression in 973 adults ages 22-78 with T2DM to be $70.7 \%$, while the prevalence of anxiety in the same population was $69.6 \%$ (Palizgir et al., 2013). A more recent meta-analysis looked 83,020,812 participants across 248 international studies, and found that overall $28 \%$ of adults with T2DM had a depressive disorder (Khaledi et al., 2019). This meta analysis also noted that females adults with T2DM were more 
likely to be depressed than their male counterparts $-34 \%$ v. $23 \%$ - and that depression among adults with T2DM was higher in Australia and Asia, 29\% and 32\% respectively (Khaledia et al., 2019). While the rates of co-morbid depression T2DM vary across studies, the impact of T2DM on depression is profound because hyperglycemia and hyperinsulinemia may result in an increase in inflammatory markers (Palizgir et al., 2013). These markers may create an overall pro-inflammatory state in various brain tissues, including the CNS which then may stimulate pathways that contribute to depressive symptom development (Palizgir et al. 2013). Poor management of T2DM has also been associated with hippocampus and amygdala reduction, classifying T2DM as a biological risk factor for depression (Palizgir et al. 2013). This inflammatory response connecting T2DM and depression resembles the aforementioned neuroimmune response connecting AUD and depression; this can yield a triage relationship between substance use, diabetes, and depression and ultimately the inflammatory nature of this triangle can inevitably lead to worsening of symptoms.

Furthermore, adults with T2DM have also been noted to have a high prevalence of anxiety disorders; a study looking at 3170 adults across 15 countries with T2DM found that the overall prevalence of any anxiety disorders in this population was 18\% (Chaturvedi et al., 2019). Among these anxiety disorders, it was found that generalized anxiety disorder was the most prevalent in the clinical population at $8.1 \%$, and panic disorder was the second-most prevalent at 5.1\% (Chaturvedi et al., 2019). Among these adults with T2DM, it was found that anxiety was significantly associated with presence of diabetic complications, poorer glycemic control, and longer duration of diabetes; on the inverse, anxiety in adults with T2DM was found to worsen clinical outcomes and impact quality of life (Chaturvedi et al., 2019). 
Earlier studies have evaluated the relationship between T2DM and psychiatric disorders, and again confirm the correlations between T2DM and depression and anxiety in adults (Collins et al., 2008). As the anxiety and depression symptoms in adults with T2DM was described as "considerably higher" than the general population, previous literature pushed for the integration of depression and anxiety screenings for patients with diabetes (Collins et al., 2008), however, limited progress has been made on this front.

The above correlations are relevant to SUD development in adults with T2DM as both depression and anxiety are risk factors associated with SUDs. Therefore, high prevalence of depression and anxiety in adults with T2DM may leave this clinical population especially vulnerable to the development of SUDs. Given the context that adults with T2DM often are associated with poor self-care and medical complications, psychiatric co-morbidities may only worsen clinical outcomes.

\section{Clinical Impacts of SUDs on Adults with T2DM}

When considering the clinical population of adults with T2DM, it is important to note that these patients already have a reduced life expectancy. A large cohort study suggests that the life expectancy of adults with T2DM may be reduced by as much as 5 years in men and 6 years in women compared to the general population (Wright et al., 2017), and that this population is at an increased risk of death before the median life expectancy in the presence of albuminuria and/or cardiovascular disease (Lutgers et al., 2010). Furthermore, this clinical population is already predisposed to medical complications resultant of long-term hyperglycemia, insulin resistance, and chronic inflammation (Schlienger, 1983, Litwak et al., 2013). These complications may include, but are not limited to, coronaropathy, CVA (stroke), myocardial 
infarction (MI), nephropathy and renal disease, retinopathy, macro- and microvascular complications, as well as foot complications that can lead to sepsis (Schlienger, 1983, Litwak et al., 2013).

Research demonstrates an increased risk of long-term diabetes complications and death among adults with T2DM and co-morbid NUD (Walter et al., 2017). This study highlights that alcohol and other illicit drug dependences — such as opioids and cocaine - may result in longterm diabetic complications by simply impairing the patient's ability to manage their T2DM and interfering with glucose homeostasis (Walter et al., 2017).

A recent study compiled 17 years of patient data from the MetroHealth System in Cleveland, Ohio to evaluate novel SUD-associated complications in adults with T2DM (Winhusen et al., 2019). This study looked at substance-specific complications, dividing the sample population into following groups: TUD, AUD, OUD, and CUD. Expanding upon previous findings, this study revealed that adults with T2DM and TUD experienced significantly higher rates of CVA, neuropathy, diabetic renal disease, MI, and death (Winhusen et al, 2019). AUD was found to increase the chances of developing diabetic neuropathy, MI, and death among adults with T2DM (Winhusen et al, 2019). These AUD-associated complications should be considered in the context that alcohol consumption in adults with T2DM can also lead to increased cardiovascular events in general and microvascular complications, as compared to adults with T2DM that do not drink (Blomster et al., 2014).

Novel research on the association of T2DM and OUD found that there was an increased risk of diabetic renal disease, $\mathrm{CVA}$, and $\mathrm{MI}$ - with hypertension as a prominent risk factor (Winhusen et al, 2019). In particular, the increased risk of renal disease is consistent with the pathology of chronic opioid use, which can result in damage to the kidneys (Winhusen et al, 
2019). Lastly, novel findings on CUD in adults with T2DM showed an increased chance of MI, diabetic neuropathy, and death; while it was noted that CUD increased the risk of CVA, this does not appear to be T2DM-specific as it is consistent with complications associated with cocaine use in the general population (Winhusen et al., 2019). With OUD and CUD in particular, this data is especially profound as the relationship between those substances and T2DM has yet to be extensively researched.

\section{Gaps in Literature}

While Winhusen et al. introduced novel research on OUD and CUD in adults with T2DM, there is still a large gap in knowledge of these specific co-morbidities. In continuation, despite TUD and AUD in diabetics having garnered more research attention, the foundation of knowledge of these SUDs leaves great room for improvement as well. Across the board, there are large discrepancies in the prevalence rates of these SUDs as well as a lack of universality in long-term complications.

Furthermore, as evidenced by cited research, there are also great discrepancies among psychiatric disorder prevalence rates, especially depression. While it is clear that all of the aforementioned disorders are highly prevalent among adults with T2DM, more consistent prevalence rates have yet to be established.

\section{Purpose}

Although research regarding co-morbid T2DM and SUDs has increased in recent years, it has only just begun to scratch the surface of this dynamic. Significant progress has been made in recognizing T2DM as a common co-morbidity associated with substance use disorders and 
T2DM is particularly well-understood with TUD and AUD (Walter et al., 2017, Winhusen et al., 2019, Wu et al., 2015). Novel research has also begun to emerge regarding T2DM and CUD as well as OUD (Winhusen et al., 2019), however, studies pertaining to those substances and their pathologies are extremely limited. It is notable that the implications of SUDs on T2DM has also gained research attention, looking at its interference with disease management as well as increase in chances of death, CVA (stroke), and MI (myocardial infarction) (Walter et al., 2017, Winhusen et al., 2019). While emerging information regarding T2DM and SUD is important, research has yet to be translated into practice effectively into relevant medical settings. Diagnostic assessments and treatment protocols specifically developed for adults with SUD and T2DM are still absent from available clinical tools, despite researchers calling for an integration of psychiatric evaluation for adults with T2DM (Wu et al. 2015, Palzigir et al. 2013, Khaledi et al., 2019, Charturvedi et al., 2019). Previous research has made it clear that there is an increased vulnerability for SUD development among adults diagnosed with T2DM, however, little has been done to address this from an assessment and intervention perspective.

The purpose of this study is to provide an educational tool to raise provider awareness of the co-morbid relationship between T2DM and SUDs. This protocol will also take into consideration psychiatric predispositions associated with T2DM, including depression and anxiety, and how they may also increase the risk of SUD development. When considering SUD prevalence and psychiatric predispositions in adults with T2DM, it becomes clear that an educational tool that will increase assessment efforts in this population is critical for their healthrelated qualify of life (HRQOL). Currently, assessment protocols and current intervention strategies for adults with T2DM and SUDs are unclear and seemingly unavailable. To address this, the proposed study aims to create and pilot an educational tool to be utilized by T2DM 
providers in order lead to the incorporation of assessment into the treatment standard for adults with T2DM.

Prior to utilizing the educational module, participating health care specialists will undergo an initial assessment to gauge their understanding of co-morbid T2DM and SUDs. Afterwards, the educational tool will then begin with a comprehensive background of the psychiatric risks associated with T2DM. This background will include some information regarding depression and anxiety, however, the primary focus will be exploring the dynamic of SUD's and T2DM. The educational module will cover prevalence rates of TUD, AUD, OUD, and CUD in adults with T2DM and observe diabetes-specific complications associated with these SUDs. This tool will then conclude with recommendations regarding implementing relevant assessments in regular treatments for those with T2DM.

Afterwards, the participating health care specialists will then take a post-education assessment to gauge their increase in awareness and understanding of co-morbid T2DM and SUDs. The overall aim of the educational module is to encourage treatment providers integrate appropriate assessments for identifying SUDs into their clinical practice. These assessments along with increased provider awareness will also assist in the treatment decision to make a psychiatric referral.

This study is based on the previous research conducted in the literature review, and seeks to answer the following research questions:

1. What is the baseline understanding endocrinology health-care providers have on T2DM and co-morbid psychiatric disorders, particularly SUDs?

2. How is research of co-morbid T2DM and SUDs currently integrated clinically? 
3. Could an educational module help improve provider understanding and recognition of co-morbid T2DM and SUDs?

\section{What T2DM-specific assessment tools can be incorporated into clinical practice to ultimately promote earlier intervention and increase HRQOL for this population?}

\section{Methodology}

\section{Research Design}

The proposed study will be a quantitative, experimental design that will utilize assigned values to gauge the effectiveness of the educational tool on co-morbid T2DM and SUDs. A preand post-educational survey will be administered to participating health care professionals to evaluate change in understanding and knowledge of co-morbid T2DM and SUDs after viewing the educational module. This study aims to specifically increase provider knowledge about comorbid TUD, AUD, OUD, and CUD in adults with T2DM and recommend assessments to implement into diabetes-related medical practices.

Participants will consist of health care professionals - predominantly physicians and nurse practitioners - working in endocrinology. Participants will be provided an initial survey to assess their baseline knowledge/education of co-morbid T2DM and SUDs. Following the initial assessment, participants will then be introduced to the educational module. This module will be a webinar-styled lecture with learning objectives pertaining to TUD, AUD, OUD, and CUD in adults with T2DM. The educational webinar will begin with relevant definitions and psychiatric risk factor statistics among adults with T2DM. The webinar will then move into SUD prevalence rates and discuss long-term, diabetes-specific complications associated with substance use. Following health care complications, the webinar will then lastly review 
appropriate assessment tools that can be implemented into the providers' endocrinology practices to promote earlier intervention. After the completion of the educational module, participants will be given a post-educational survey to evaluate the efficacy of the educational webinar in increasing knowledge/education of co-morbid T2DM and TUD, AUD, OUD, and CUD. Survey responses will be scored and a greater numerical difference between the pre- and posteducational surveys will reflect a greater change in knowledge of co-morbid T2DM and the aforementioned SUDs.

\section{Sampling and Setting}

The sample of this study will primarily consist of physicians and APRNs practicing in the field of endocrinology, the predominant healthcare specialists that work with T2DM. However, additional healthcare providers, such as a physician assistant (PA) or primary care physician, may participate in the study provided that they are the primary medical provider to T2DM-heavy populations. This study is also open to internal medicine residents pursuing an endocrinology fellowship or current endocrinology fellows, as their work and prospective patients consists of adults with T2DM. Providers will be eligible if they fulfill the following inclusion criteria:

1.) Currently practicing clinician with possession of appropriate medical licensure or certification, such as a MD, DO, PA, APRN.

2.) Health care professional heavily working with T2DM populations or with the intent to work with this population, OR mental health and/or treatment providers treating clients with T2DM.

This study also implements the following exclusion criteria: 
1.) Healthcare professionals in specialties that do not pertain to T2DM care (such as orthopedics).

2.) Professionals that only possess non-clinical doctorates and do not work directly with patients.

3.) Professionals that do not speak or read English fluently.

4.) Professionals that are not at least 21 years of age.

5.) Professionals that are retired.

Study participants will be recruited from their current places of employment, with a specific recruitment focus on outpatient endocrinology clinics. Due to the virtual nature of this study, participants will be recruited from across the United States and contacted via their workassociated email. The study aims to recruit approximately 50-100 providers from different regions of the United States to participate in the study. Interested health care providers will be given further information about the study and are required to provide written informed consent before study participation.

\section{Procedure}

After approval by WCSU IRB, the primary researcher of this study will administer a 20item pre-educational questionnaire to study participants to evaluate initial education of comorbid T2DM and TUD, AUD, OUD, and/or CUD, as well as the associated psychiatric risk factors.

Study participants will then view the educational webinar, which will be structured based on the following outline:

1.) Learning objectives 
2.) Overview: important psychiatric risk factor considerations

3.) Definitions and criteria: TUD, AUD, OUD, CUD, and associated terminology

4.) Prevalence Rates: SUDs in T2DM compared to the general population

5.) Long-term, diabetes-specific SUD complications: T2DM and TUD, AUD, OUD, and CUD

6.) Assessment Instruments: Beck Depression and Anxiety Inventories, Alcohol Use Disorders Identification Test (AUDIT), and original TUD, OUD, \& CUD screening

The webinar will emphasize the following learning objectives for the study participants:

1.) Goal: increase provider knowledge about SUD development risk associated with T2DM.

2.) Goal: provide prevalence statistics on T2DM and AUD, TUD, OUD, and CUD.

3.) Goal: increase knowledge of long-term, diabetes-specific health complications that may arise from the aforementioned substance use.

4.) Goal: have access to appropriate TUD, AUD, OUD, and CUD assessment tools to incorporate into diabetes-related practice.

5.) Goal: educate providers on when a referral for substance treatment is appropriate to achieve adequate interdisciplinary care

After participants complete the educational module and fulfill the learning objectives, a posteducational survey will then be administered. This survey will consist of 20 questions to evaluate participant's new level of education on T2DM and SUDs following use of the webinar.

\section{Measurements}

Both pre- and post-educational surveys will be answered using a Likert scale of 1-5. The numbers will represent the following answers: $1=$ no understanding, $2=$ minimal understanding, 
$3=$ some understanding, $4=$ good understanding, $5=$ expert understanding. The surveys will then be scored by adding up the participant's answers; a positive change in points from pre- to post-educational survey will reflect greater education on co-morbid T2DM and SUDs.

\section{Data Analysis}

For comparative purposes, the average change in participant education will then be converted and expressed in a percentage, using the following calculation:

[(Avg. Post-Edu. Score - Avg. Pre-Edu. Score) / Avg. Pre-Edu. Score] $* 100=\%$ change in education A Student's T-test will also be conducted to compare the means of the pre-educational assessment score to the post-educational assessment score. This specific test is being used to determine if the difference in mean scores, and therefore difference in education, is statistically significant. The cut-off $\mathrm{P}$ value will be set at 0.05 , with results exceeding a $\mathrm{P}$-value of 0.05 indicating no statistically significant difference between the means. A P-value of $\leq 0.05$ will indicate a statistically significant difference between the means, which supports that the educational module was efficacious and increased provider education on co-morbid T2DM and SUDs. This increase in provider education will then hopefully yield the widespread implementation of appropriate assessment tools in T2DM-related settings. 


\section{Sample: Pre-Education Survey}

The following survey uses a Likert Scale. Questions will be answered with a number system ranging from 1-5, where: $1=$ no understanding, $2=$ minimal understanding, $3=$ some understanding, $4=$ good understanding, and $5=$ expert understanding. Please review the values and their associated meanings thoroughly before answering the survey questions. The survey should be answered as honestly as possible.

\begin{tabular}{|c|c|c|c|c|c|}
\hline Question & 1 & 2 & 3 & 4 & 5 \\
\hline $\begin{array}{l}\text { 1. Type } 2 \text { diabetes is a condition that has health } \\
\text { implications outside of the endocrine system. }\end{array}$ & & & & & \\
\hline $\begin{array}{l}\text { 2. Type } 2 \text { diabetes is a biological risk factor for } \\
\text { depression. }\end{array}$ & & & & & \\
\hline $\begin{array}{l}\text { 3. Prevalence of depression is higher in type } 2 \\
\text { diabetics than the general population. }\end{array}$ & & & & & \\
\hline $\begin{array}{l}\text { 4. Prevalence of anxiety is higher in type } 2 \\
\text { diabetics than the general population. }\end{array}$ & & & & & \\
\hline $\begin{array}{l}\text { 5. Psychiatric co-morbidities associated with type } \\
2 \text { diabetes leaves this population susceptible to } \\
\text { developing substance use disorders. }\end{array}$ & & & & & \\
\hline $\begin{array}{l}\text { 6. Any substance use in adults with type } 2 \text { diabetes } \\
\text { may interfere with glucose homeostasis and } \\
\text { disease management ability. }\end{array}$ & & & & & \\
\hline $\begin{array}{l}\text { 7. Tobacco use disorder, which may involve } \\
\text { cigarettes and/or chewing tobacco, is the most } \\
\text { common substance use disorder in adult } \\
\text { diabetics. }\end{array}$ & & & & & \\
\hline $\begin{array}{l}\text { 8. Tobacco use disorder in adults with type } 2 \\
\text { diabetes increases risk of long-term } \\
\text { complications, such as neuropathy and renal } \\
\text { disease. }\end{array}$ & & & & & \\
\hline $\begin{array}{l}\text { 9. Alcohol use disorder is more prevalent in adults } \\
\text { with type } 2 \text { diabetes than the general population. }\end{array}$ & & & & & \\
\hline $\begin{array}{l}\text { 10. Non-diabetic patients with alcohol use disorder } \\
\text { are at greater risk for developing type } 2 \\
\text { diabetes. }\end{array}$ & & & & & \\
\hline
\end{tabular}




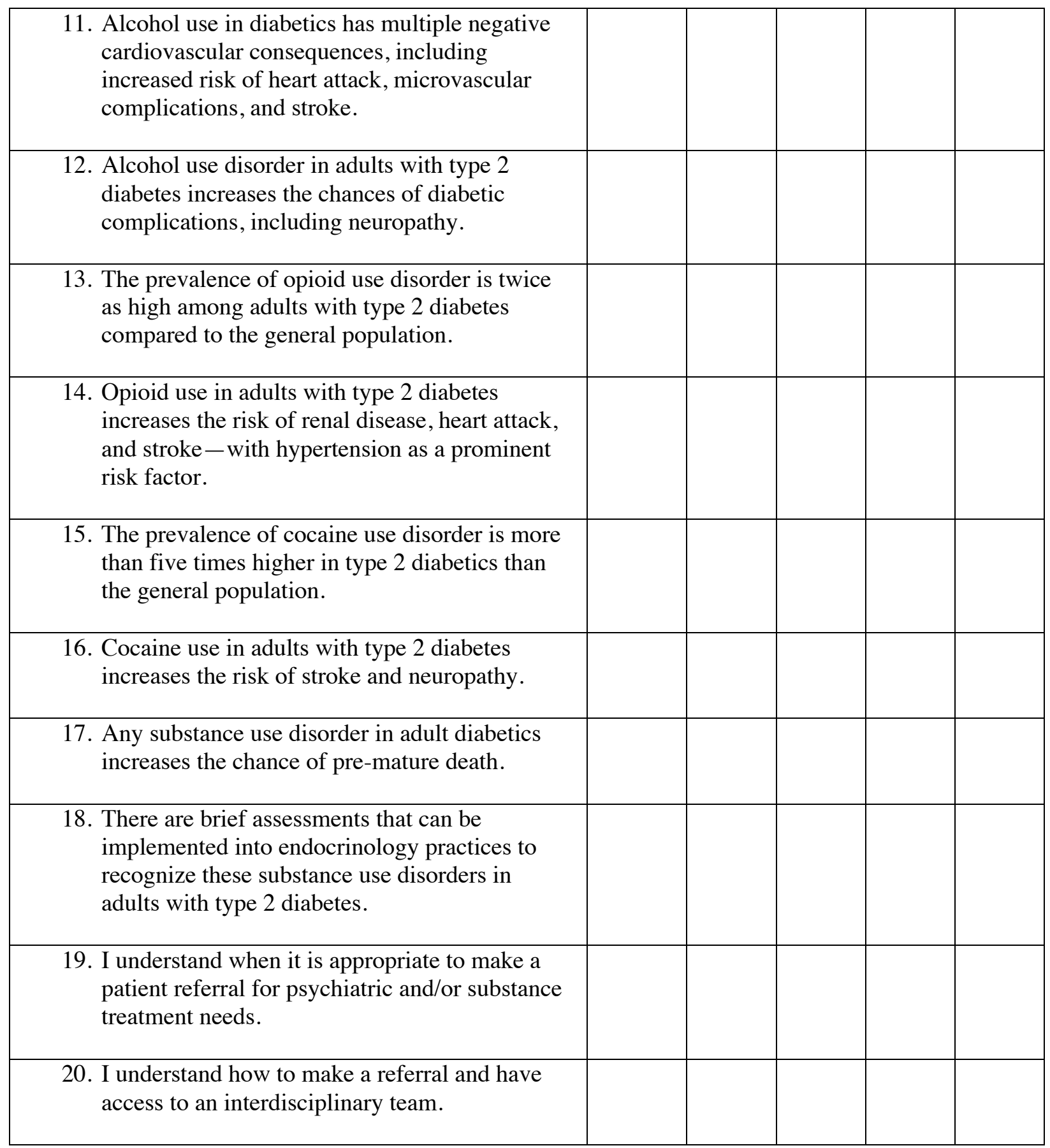

Score: 


\section{Sample: Post-Education Survey}

The following survey uses a Likert Scale. Questions will be answered with a number system ranging from 1-5, where: 1 = no understanding, $2=$ minimal understanding, $3=$ some understanding, $4=$ good understanding, and $5=$ expert understanding. Please review the values and their associated meanings thoroughly before answering the survey questions. The survey should be answered as honestly as possible.

\begin{tabular}{|c|c|c|c|c|c|}
\hline Question & 1 & 2 & 3 & 4 & 5 \\
\hline $\begin{array}{l}\text { 1. Type } 2 \text { diabetes is a condition that has health } \\
\text { implications outside of the endocrine system. }\end{array}$ & & & & & \\
\hline $\begin{array}{l}\text { 2. Type } 2 \text { diabetes is a biological risk factor for } \\
\text { depression. }\end{array}$ & & & & & \\
\hline $\begin{array}{l}\text { 3. Prevalence of depression is higher in type } 2 \\
\text { diabetics than the general population. }\end{array}$ & & & & & \\
\hline $\begin{array}{l}\text { 4. Prevalence of anxiety is higher in type } 2 \\
\text { diabetics than the general population. }\end{array}$ & & & & & \\
\hline $\begin{array}{l}\text { 5. Psychiatric co-morbidities associated with type } \\
2 \text { diabetes leaves this population susceptible to } \\
\text { developing substance use disorders. }\end{array}$ & & & & & \\
\hline $\begin{array}{l}\text { 6. Any substance use in adults with type } 2 \text { diabetes } \\
\text { may interfere with glucose homeostasis and } \\
\text { disease management ability. }\end{array}$ & & & & & \\
\hline $\begin{array}{l}\text { 7. Tobacco use disorder, which may involve } \\
\text { cigarettes and/or chewing tobacco, is the most } \\
\text { common substance use disorder in adult } \\
\text { diabetics. }\end{array}$ & & & & & \\
\hline $\begin{array}{l}\text { 8. Tobacco use disorder in adults with type } 2 \\
\text { diabetes increases risk of long-term } \\
\text { complications, such as neuropathy and renal } \\
\text { disease. }\end{array}$ & & & & & \\
\hline $\begin{array}{l}\text { 9. Alcohol use disorder is more prevalent in adults } \\
\text { with type } 2 \text { diabetes than the general population. }\end{array}$ & & & & & \\
\hline $\begin{array}{l}\text { 10. Non-diabetic patients with alcohol use disorder } \\
\text { are at greater risk for developing type } 2 \\
\text { diabetes. }\end{array}$ & & & & & \\
\hline
\end{tabular}




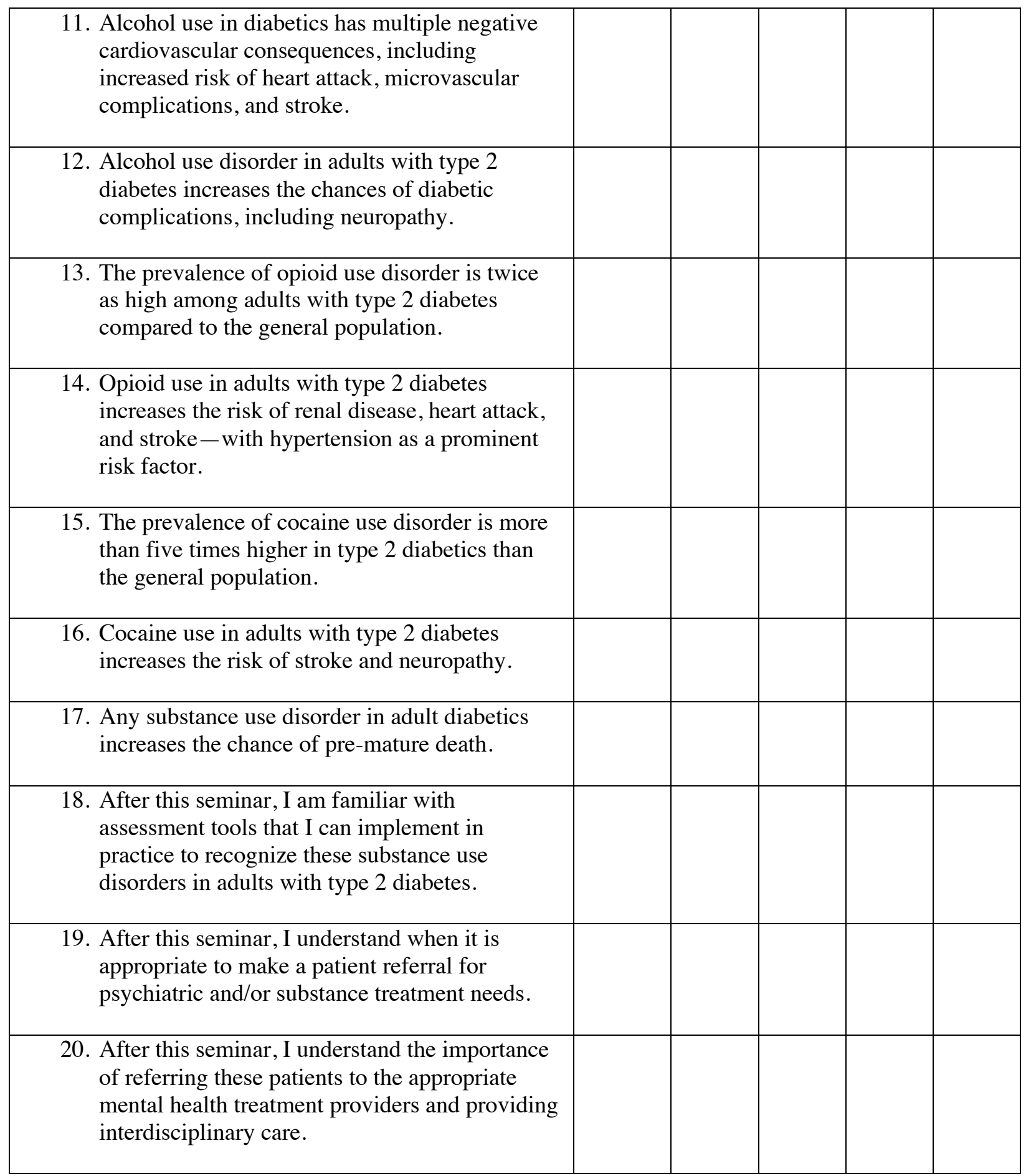

Score: 


\section{Sample: Original Tobacco Use Disorder Screening}

1. How frequently do you use tobacco products? Please circle the closest answer:

Never $1 \mathrm{x} /$ year $1 \mathrm{x} /$ month $1 \mathrm{x} /$ week Daily

2. How many years have you been using tobacco products for? Please circle the closest answer:

0 years 1-2 years 3-4 years 5-6 years 6+ years

3. How many packs of cigarettes or containers of chewing tobacco do you use in a week? Please circle the closest answer:

0 packages 1-2 packages 3-4 packages 5-6 packages 6+ packages

4. If you do consume tobacco currently, have you ever successfully quit? Please circle one answer:

YES NO N/A

5. If you do consume tobacco currently, do you have any desire to quit? Please circle one answer:

YES NO N/A

**Providers:

For an answer indicating monthly use or greater for question 1, please ask your patient further about their tobacco use habits. For any answer greater than 0 for questions 2-3 or any answers "yes" on questions 4-5, please speak to your patient about their tobacco use and consider making a referral. 


\section{Sample: Original Opioid Use Disorder Screening}

1. What is your lifetime use of opioids? Please circle one answer:

0 times $\quad$ 1-2 times $\quad 3-4$ times $\quad 5-6$ times $\quad 6+$ times

2. In the past 6 months, what has been your use of opioids? Please circle one answer:

0 times $\quad$ 1-2 times $\quad 3-4$ times $\quad 5-6$ times $\quad 6+$ times

3. Have you ever consumed opioids that were not prescribed to you? Please circle one answer:

YES NO

4. Have you ever taken opioids recreationally? Please circle one answer:

YES NO

5. Have you ever taken above the prescribed amount of opioids? Please circle one answer:

YES NO

6. Have you ever overdosed and/or been hospitalized for opioid use? Please circle one answer:

YES NO

**Providers:

For any answer greater than 0 for questions $1 \& 2$, please ask your patient further about the circumstances of their opioid use habits. For any answers "yes" to questions 3-6, please discuss opioid use further with your patient and consider making a referral. 


\section{Sample: Original Cocaine Use Disrder Screening}

1. What is your lifetime use of cocaine? Please circle one answer:

0 times $\quad$ 1-2 times 3-4 times 5-6 times $\quad 6+$ times

2. In the past 6 months, what has been your use of cocaine? Please circle one answer:

0 times 1-2 times 3-4 times 5-6 times $\quad 6+$ times

3. If you currently consume cocaine, have you ever successfully quit? Please circle one answer:

YES NO N/A

4. If you currently consume cocaine, do you have any desire to quit? Please circle one answer:

YES NO N/A

5. Have you ever overdosed and/or been hospitalized for cocaine use? Please circle one answer:

YES NO N/A

**Providers:

For any answer greater than 0 for questions $1 \& 2$, please ask your patient further about their cocaine use. For any answers "yes" or "no" to questions 3-4 or "yes" to question 5, please discuss opioid use further with your patient and consider making a referral. 


\section{Projected Impact}

\section{Clinical Implications}

Information covered in the scope of this study is critical for clinicians specializing in T2DM care as well as mental health providers working with adults with T2DM. The information from this study will bring attention to the lack of education on co-morbid T2DM and TUD, AUD, OUD, and CUD. Furthermore, this study aims to inform and supplement this missing foundation by allowing participants to go through an educational module on the aforementioned SUDs in adults with T2DM and reviewing the psychiatric risk factors. The change in mean scores from pre- to post-educational survey will reflect increase in provider education and awareness of the need for interdisciplinary based care, with the implementation of appropriate SUD and psychiatric assessments into regular endocrinology practices.

This research specifically assists with substance use education in health care professionals working with diabetics by providing them with a webinar to fortify their education on co-morbid T2DM and TUD, AUD, OUD, and CUD. Additionally, this research plays an important role in substance use assessment by introducing providers to relevant assessment tools, such as Beck Inventories, AUDIT, as well as original TUD, OUD, and CUD screenings. While this research does not directly delve into the treatment or prevention of co-morbid T2DM and SUDs, the implementation of appropriate assessment tools at regular T2DM visits promotes earlier substance use intervention and encourages the formation of an interdisciplinary care team for this clinical population.

The results of this research can assist clinicians to better serve patients again by promoting interdisciplinary psychiatric and endocrinologic care to adults with T2DM. By 
enabling endocrinologists and health care specialists that work with T2DM populations to recognize and assess substance use, patients with T2DM will receive mental health intervention and substance use treatments sooner if deemed appropriate. This will ultimately yield an improvement in clinical outcomes for these T2DM patients as well as an increase in the HRQOL.

The participation of mental health treatment providers in this study is also crucial for providing adults with T2DM adequate care. The information provided by the educational module can inform case concepts and treatment plans conducted by the mental health providers. By understanding T2DM better, these mental health treatment providers can gain further insight on client background. In continuation, this study can encourage mental health treatment providers to integrate a component of psychoeducation into the treatment plans of those with T2DM. This psychoeducation can inform their diabetic clients on the increased risk of substance use associated with T2DM as well as the long-term complications of co-morbid T2DM and SUDs.

Outside of substance use and mental health treatment providers, clinical providers working in endocrinology with T2DM adults would benefit greatly from the knowledge produced by this research. As previously mentioned, these T2DM clinical providers - such as physicians, nurse practitioners, and physician assistant - can significantly further their education on co-morbid T2DM and SUDs and help with earlier recognition of SUDs by implementing the appropriate assessment tools.

\section{Individual Impact}

This research can benefit consumers of both endocrinologic and behavioral health care. Oftentimes, adults seeking treatment for T2DM fail to have their co-morbidities recognized by health care providers. By fortifying provider education on T2DM and SUDs and implementing 
relevant assessment tools into regular diabetes visits, this clinical population can expect earlier interventions which could improve HRQOL and prevent long-term complications associated with these co-morbidities.

\section{Community Impact}

This research also can benefit the larger community of individuals with co-morbid T2DM and SUDs. The formation of support groups for these co-morbidities may arise, as more patients are recognized and treated for T2DM and SUDs. Additionally, this research could encourage the implementation of more education on T2DM and TUD, AUD, OUD, and CUD in medical curriculums, in particular medical schools, PA schools, as well as nursing programs.

\section{Policy Impact}

When considering this research, federal policy recommendations regarding medical licensure can require annual or bi-annual trainings on T2DM and TUD, AUD, OUD, and CUD. As the prominence of T2DM rises alongside the growing awareness of SUDs, policies inclusive of trainings on both should be pushed for by the federal government.

Furthermore, implementing these trainings as a part of medical licensure policies may be economically beneficial in the long-run. By educating health care providers and implementing mental health and substance assessments into practice, the costs associated with long-term T2DM complications may be reduced. By making substance use treatment referrals earlier, there may also be a reduction in cost of substance treatment because earlier recognition and intervention is crucial for reducing risk of relapse. 


\section{$\underline{\text { Conclusions }}$}

\section{Future Directions}

Following this study, there are several avenues of research that may be pursued in the future to build upon the information acquired here. Firstly, the study could be expanded to a larger number of participating clinicians. The initial study proposed using 50-100 participants from various parts of the United States, however, this may not be sufficient enough to be a reliable, representative sample. Future research could involve repeating this process and educational module with a greater number of participants - with a goal to exceed $n=1,000$.

Secondly, while this study encompasses tobacco, alcohol, opioids, and cocaine, further research could be done with substances outside of this realm. This includes - but is not limited to- marijuana use in adults with T2DM. Associated risk factors, prevalence rates of cannabis use disorder, and long-term complications associated with cannabis use in adults with T2DM could be studied further and integrated into the educational webinar.

Lastly, the primary focus of this study was adults with type 2 diabetes, however, it is important to note that there are many other forms of diabetes as well, such as type 1 diabetes mellitus (T1DM). Future research could look for correlations between SUDs and T1DM, and explore any associated complications that may be T1DM-specific. This also opens up the opportunity to compare risk of SUD development between different types of diabetes, such as a comparison of SUD prevalence rates in T2DM v. T1DM.

\section{Conclusion}

In reiteration, health care providers working with T2DM clinical populations are missing important education on the co-morbid dynamic of T2DM and TUD, AUD, OUD, and CUD. 
While research in this field is limited and poses various prevalence rates, it has become apparent that this co-morbid dynamic is serious and needs to be addressed. In particular, OUD and CUD have lacked significant research attention in terms of prevalence, and across the board there is a need for more information and resources regarding the long-term complications associated with substance use and T2DM. The aim of the present study was to bridge some of the gaps in literature and improve the education that providers have on T2DM and co-morbid TUD, AUD, OUD, and CUD. Implications of the present study include highlighting the need for provider education on substance use in adults with T2DM, as well as the implementation of relevant assessment tools in the clinical setting. 


\section{$\underline{\text { References }}$}

American Psychiatric Association. (2013). Diagnositc and statistical mangual of mental disorders ( $\left.5^{\text {th }} \mathrm{ed}.\right)$.

Blomster, J., S. Zoungas, J. Chalmers, Q. Li, C. Chow, M. Woodward... G. Hills. (2014). The relationship between alcohol consumption and vascular complications and mortality in individuals with type 2 diabetes. Diabetes Care 37: 1353-1359.

Chaturvedi, S., S. Gowda, H. Ahmed, F. Alosaimi, N. Andreone, A. Bobrov... N. Sartorius. (2019). More anxious than depressed: prevalence and correlates in a 15-nation study of anxiety disorders in people with type 2 diabetes mellitus. General Psychiatry 32: e100076. Doi: 10.1136/gpsych-2019-100076.

Collins, M., P. Corcoran, and I. Perry. (2008). Anxiety and depression symptoms in patients with diabetes. Diabetic Medicine 26: 153-161.

Hunt, G., G. Malhi, H. Man Xiong Lai, and M. Cleary. (2020). Prevalence of comorbid substance use in major depressive disorder in community and clinical settings, 19902019: systematic review and meta-analysis. Journal of Affective Disorders 266: 288-304.

Iqbal, M., C. Levin, and F. Levin. (2019). Treatment for substance use disorder with cooccurring mental illness. Focus 17: n.p. 
Khaledi, M., F. Haghighatdoost, A. Feizi, and A. Aminorroaya. (2019). The prevalence of comorbid depression in patients with type 2 diabetes: an unpdated systematic review and meta-analysis on huge number of observational studies. Acta Diabetologica 56: 631-650.

Neupane, S. (2016). Neuroimmune interface in the comorbidity between alcohol use disorder and major depression. Front. Immunol. 7:655. Doi: 10.3389/fimmu.2016.00655

Litwak, L., S. Goh, Z. Hussein, R. Malek, V. Prusty, and M. Khamseh. (2013). Prevalence of diabetes complications in people with type 2 diabetes mellitus and its association with baseline characteristics in the multinational $A_{l}$ Chieve Study. Diabetology \& Metabolic Syndrome 5: n.p. Doi: 10.1186/1758-5996-5-57.

Lipari, R. and S. Van Horn. (2017). Trends in substance use disorders among adults aged 18 or older. The CBHSQ Report: June 29, 2017. Center for Behavioral Health Statistics and Quality, Substance Abuse and Mental Health Services Administration, Rockville, MD.

Lutgers, H., E. Gerrits, W. Sluiter, L. Ubink-Veltmaat, G. Landman, T. Links, R. Gans, A. Smit, and H. Bilo. (2009). Life expectancy in a large cohort of type 2 diabetes patients treated in primary care (ZODIAC-10). PLoS ONE 4: e6817.

Palizgir, M., M. Bakhtiari, and A. Esteghamati. (2013). Association of depression and anxiety with diabetes mellitus type 2 concerning some sociological factors. Iran Red Crescent Med J 15: 644-648. 
Schlienger, JL. (1983). Type 2 diabetes complications. Presse Medicale 42: 839-848.

Smith, J. and S. Book. (2008). Anxiety and substance use disorders: a review. Psychiatr Times 25: $19-23$.

Vancampfort, D., J. Mugisha, M. Hallgren, M. De Hert, M. Probst, D. Monsieur, and B. Stubbs. (2016). The prevalence of diabetes mellitus type 2 in people with alcohol use disorders: a systematic review and large scale meta-analysis. Psychiatry Research 246: 394-400.

Walter, K., J. Wagner, E. Cengiz, W. Tamborlane, and N. Petry. (2017). Substance use disorders among patients with type 2 diabetes: a dangerous but understudied combination. Curr. Diab. Rep. 17: n.p.

Winhusen, T., J. Theobald, D. Kaelber, and D. Lewis. (2019). Medical complications associated with substance use disorders in patients with type 2 diabetes and hypertension: electronic health record findings. Addiction 114: 1462 - 1470.

Wells, K., J. Golding, and A. Burnam. (1989). Affective, substance use, and anxiety disorders in persons with arthritis, diabetes, heart disease, high blood pressure, or chronic lung conditions. General Hospital Psychiatry 11: 320-327. 
Wright, A., E. Kontopantelis, R. Emsley, I. Buchan, N. Sattar, M. Rutter, and D. Ashcroft. (2017). Life expectancy and cause-specific mortality in type 2 diabetes: a populationbased cohort study quantifying relationships in ethnic subgroups. Diabetes Care 40: 338345.

Wu, L., U. Ghitza, B. Batch, M. Pencina, L. Rojas, B. Goldstein, T. Schibler, A. Dunham, S. Rusincovitch, and K. Brady. (2015). Substance use and mental diagnoses among adults with and without type 2 diabetes: results from electronic health records data. Drug and Alcohol Dependence 156: 162-169. 